\title{
Changes in Red Blood Cell Behavior during Cerebral Blood Flow Increase in the Rat Somatosensory Cortex: A Study of Laser-Doppler Flowmetry
}

\author{
Tetsuya MATSUURA and Iwao KANNO \\ Department of Radiology and Nuclear Medicine, Akita Research Institute of \\ Brain and Blood Vessels, Akita, 010-0874 Japan
}

\begin{abstract}
The purpose of this study was to investigate red blood cell (RBC) behavior during an increase in local cerebral blood flow (LCBF). We measured changes in RBC behavior by using laser-Doppler flowmetry (LDF) in $\alpha$-chloralose-anesthetized rats. An increase in LCBF was carried out by approximately 2.5 and $4.0 \%$ $\mathrm{CO}_{2}$ inhalation and activation of the somatosensory cortex. The activation of the cortex was induced by electrical stimulation of the hind paw with $1.5-\mathrm{mA}$ pulses $(0.1 \mathrm{~ms})$ applied at frequencies of $0.2,1,5$, and $10 \mathrm{~Hz}$ for a $5 \mathrm{~s}$ duration. The increases in LCBF and RBC velocity during both $\mathrm{CO}_{2}$ inhalations were larger than that in $\mathrm{RBC}$ concentration $(p<0.05)$. LCBF and RBC velocity during $4.0 \% \quad \mathrm{CO}_{2}$ inhalation were larger than those during $2.5 \% \quad \mathrm{CO}_{2}$ inhalation $(p<0.05)$,
\end{abstract}

though there was no significant difference in RBC concentration between the two conditions, suggesting a limitation of capillary volume. During somatosensory stimulation, the evoked LCBF increased with increasing stimulus frequency up to $5 \mathrm{~Hz}$ and decreased at $10 \mathrm{~Hz}$. The responses of RBC concentration at 0.2 and $10 \mathrm{~Hz}$ were greater than those of RBC velocity $(p<0.05)$, but no significant differences in response magnitude were found at 1 and $5 \mathrm{~Hz}$ between RBC concentration and $R B C$ velocity. These results suggest that the increase in LCBF during neuronal activity is different from that of controlling the LCBF as induced by $\mathrm{CO}_{2}$, and that the regulation of RBC concentration and RBC velocity is controlled by independent mechanisms. [Japanese Journal of Physiology, 51, 703-708, 2001]

Key words: cerebral blood flow, hypercapnia, laser-Doppler flowmetry, somatosensory stimulation, rat.

It has been accepted that the change in local cerebral blood flow (LCBF) induced by sensory stimulation (evoked LCBF) reflects a neuronal activity in the brain, since the coupling between LCBF and neuronal activity was first proposed by Roy and Sherrington [1]. However, the mechanisms underlying evoked LCBF remain to be clarified because the relationship among neuronal activation, metabolism, and change in LCBF is highly complex. Several factors, such as metabolic by-products (e.g., proton, potassium, adenosine, and nitric oxide) and neurogenic control, might be responsible for the vessel diameter change in neurovascular coupling on cortical activation [2]. Oxygen and carbon dioxide may also be involved in LCBF regulation during neuronal activity [3, 4]. It is well known that the $\mathrm{CO}_{2}$ reactivity is mediated by $\mathrm{pH}$ variations around the arteriolar site (resistance vessel), and the $\mathrm{pH}$ variations influence the tone of the vascular smooth muscle [5]. This indicates that a change in $\mathrm{PaCO}_{2}$ level is associated with the dilation of resistance vessels, causing a change in precapillary pressure. The location of vasculature, however, where microcirculation is regulated during neuronal activity, has been poorly understood. Some investigators observed that the diameter of the pial arteriole reflects the amount of blood flow during neuronal activation

Received on May 28, 2001; accepted on September 4, 2001

Correspondence should be addressed to: Tetsuya Matsuura, Department of Biology and Earth Sciences, Faculty of Science, Ehime University, Bunkyo-cho 3, Matsuyama, 790-8577 Japan. Tel and Fax: +81-89-927-8928, E-mail: matsuurat@sci.ehime-u.ac.jp 
of the cortex [6]. Malonek et al. noted in the cat visual cortex that the cerebral blood volume increase preceded the CBF increase and assumed that the capillary change occurs before arteriolar dilation [7]. In our previous studies, we hypothesized that the capillary and the resistance vessels both contribute to the regulation of evoked LCBF $[8,9]$, and the regulation by these vessels is controlled by independent mechanisms [8].

The purpose of this study was to test the above hypothesis by measuring the change in red blood cell (RBC) behavior, using laser-Doppler flowmetry (LDF), during the increase in LCBF. LDF has been used to monitor cerebral microcirculation in the capillary bed. Several laboratories have used LDF to demonstrate LCBF changes related to neuronal activation by various stimuli $[3,4,6,8-13]$. LDF has also been improved to monitor $\mathrm{RBC}$ velocity and $\mathrm{RBC}$ concentration independently, and the validations of these parameters were achieved by in vitro experiments [10]. It was considered that RBC behavior in the capillary bed is the main contributor to the LDF signal [10], suggesting that the vascular behavior of the capillary and resistance vessel during LCBF increase is indirectly determined from the change in $\mathrm{RBC}$ concentration or RBC velocity [3, 4, 8-11]. In the present study, we examined the response of LCBF, $\mathrm{RBC}$ concentration, and $\mathrm{RBC}$ velocity during various levels of the somatosensory activation and $\mathrm{CO}_{2}$ inhalation (hypercapnia) to investigate vascular behavior.

\section{MATERIALS AND METHODS}

Sprague-Dawley rats $(330-420 \mathrm{~g})$, which were bred in a closed colony (Charles River Japan Inc., Japan), were anesthetized with halothane (4\% for induction and $1.5 \%$ during surgery) in $30 \%$ oxygen and $70 \%$ nitrous oxide. The tail artery and the left femoral vein were cannulated for blood pressure monitoring, blood gas sampling, and intravenous drug administration. Following tracheotomy, $\alpha$-chloralose $(75 \mathrm{mg} / \mathrm{kg}, \mathrm{I.V}$.) was administered, and halothane and nitrous oxide administrations were discontinued. The rat was immobilized with pancuronium bromide $(0.7 \mathrm{mg} / \mathrm{kg}$, I.V. $)$ and ventilated with a respirator (SN-480-7, Shinano, Japan) using room air and supplemental oxygen throughout the experimental period. Anesthesia was maintained with $\alpha$-chloralose $(45 \mathrm{mg} / \mathrm{kg} / \mathrm{h}, \mathrm{I}$.V.), and muscle relaxation with pancuronium bromide $(0.8$ $\mathrm{mg} / \mathrm{kg} / \mathrm{h}$, I.V.). The body temperature was maintained at approximately $37.0^{\circ} \mathrm{C}$ by use of a heating pad (ATC-101, Unique Medical, Japan). The rat was fixed in a stereotactic frame and the parietal bone was thinned to translucency at the left somatosensory cortex over an area of $3 \times 3 \mathrm{~mm}^{2}$, centered at $2.5 \mathrm{~mm}$ caudal and $2.5 \mathrm{~mm}$ lateral to the bregma. The $\mathrm{PaCO}_{2}$ levels were maintained in a range of $32-40 \mathrm{mmHg}$ and the $\mathrm{PaO}_{2}$ levels in a range of $90-120 \mathrm{mmHg}$ by regulating the stroke volume of ventilation and the fractional concentration of oxygen in the inspired gas, respectively.

LCBF was measured with an LDF (Periflux 4001 Master, Perimed, Sweden) equipped with an LDF probe with a tip diameter of $0.46 \mathrm{~mm}$ (Probe 411, Perimed). LDF measures blood flow based on the Doppler effect by using laser light. Our LDF system simultaneously provides three parameters: flux (=LCBF), RBC concentration, and $\mathrm{RBC}$ velocity, where $\mathrm{RBC}$ velocity $=\mathrm{LCBF} / \mathrm{RBC}$ concentration [14]. The volume of LDF measurement was about $1 \mathrm{~mm}^{3}$. A time constant of $0.03 \mathrm{~s}$ was used for measuring all LDF signals (LCBF, RBC concentration, and RBC velocity). The LDF probe was placed in the somatosensory area of the hind paw, perpendicular to the brain surface. It was attached to the thinned parietal bone, avoiding areas with large blood vessels.

To ensure a stable condition of the animal, measurements were performed $2-3 \mathrm{~h}$ after the preparation of the parietal bone. An induction of the increase in LCBF was carried out by $\mathrm{CO}_{2}$ inhalation (Study 1) and somatosensory stimulation (Study 2). In Study 1, $\mathrm{PaCO}_{2}, \mathrm{LCBF}, \mathrm{RBC}$ concentration, and $\mathrm{RBC}$ velocity were successively measured after approximately 2.5 and $4.0 \% \mathrm{CO}_{2}$ inhalation $(n=7)$. This paradigm was to investigate the effect of hypercapnia on hemodynamic responses. We first examined hemodynamic response under $2.5 \% \mathrm{CO}_{2}$ inhalation, followed by that under $4.0 \% \mathrm{CO}_{2}$ inhalation after $2 \mathrm{~h}$ of offset of $2.5 \%$ $\mathrm{CO}_{2}$ inhalation. In Study 2, the activation of the cortex was carried out by electrical pulse stimulation of the hind paw (pulse width of $0.1 \mathrm{~ms}$ ), using a pair of small needle electrodes inserted under the skin of the right hind paw $(n=14)$. The frequencies of $0.2,1,5$, and $10 \mathrm{~Hz}$ of electrical stimuli were applied for a $5 \mathrm{~s}$ duration at an intensity of $1.5 \mathrm{~mA}$. The order of the stimulus frequencies was selected randomly; at each stimulus frequency, 30 successive stimuli were applied at $60 \mathrm{~s}$ intervals.

The LDF signals and arterial blood pressure were recorded continuously by using the MacLab data-acquisition software (AD Instruments, Australia). LDF data of 30 successive measurements in Study 2 were accumulated to reduce the noise level of the LDF signal and saved on a disk for off-line analyses. The LDF data were normalized to the prestimulus value (base- 
line level). In Study 2, the rise time, peak time, and termination time of the evoked LCBF response were measured as a function of stimulus frequency. The rise time of the LDF signal was determined as the intersection of the baseline and the extrapolated line, which was drawn on the normalized response curve from 90 to $10 \%$ of the peak, and the termination time to the baseline level was determined as the time at the intersection of the baseline by a similar extrapolated line (detail in Matsuura et al. [8]). The peak time was when the response curve reached maximum height. The response magnitude for the $5 \mathrm{~s}$ hind-paw stimulation was calculated as the integral of the normalized response curve from the rise time to the termination time and was considered to reflect the total increase in blood flow.

Parameters among each stimulus frequency were statistically analyzed by ANOVA (repeated measurements) and multiple comparisons (Bonferroni). Values are presented as means \pm SD. All experiments were in accordance with the guidelines of the Physiological Society of Japan and were approved by the Animal Care and Use Committee of Akita Research Institute of Brain and Blood Vessels.

\section{RESULTS}

\section{Changes in RBC behavior under hypercapnic condition (Study 1)}

Hypercapnia, which was induced by adding approximately 2.5 and $4.0 \% \mathrm{CO}_{2}$, increased the $\mathrm{PaCO}_{2}$ level and LDF parameters gradually with a slight decrease in the mean arterial blood pressure (MABP). The MABP after $30 \mathrm{~min}$ of 2.5 and $4.0 \% \mathrm{CO}_{2}$ inhalation decreased to $89.5 \pm 7.2 \mathrm{mmHg}$, from 101.4 $\pm 8.4(p<$ $0.05)$, and to $90.1 \pm 7.2 \mathrm{mmHg}$, from $100.7 \pm 7.2(p<$ $0.05)$, respectively. There was no significant difference in $\mathrm{PaO}_{2}$ level between normocapnia and hypercapnia. $\mathrm{PaO}_{2}$ levels after $30 \mathrm{~min}$ of 2.5 and $4.0 \% \mathrm{CO}_{2}$ inhalation were $101.9 \pm 6.0$ and $100.5 \pm 11.9 \mathrm{mmHg}$, respectively $(105.6 \pm 7.5 \mathrm{mmHg}$ under normocapnia).

The $\mathrm{PaCO}_{2}$ level, LCBF, and RBC velocity reached peak values after approximately $20 \mathrm{~min}$ of both levels of $\mathrm{CO}_{2}$ inhalation (Fig. 1). RBC concentration reached a peak value after approximately $15 \mathrm{~min}$ of $\mathrm{CO}_{2}$ inhalation (Fig. 1). After $20 \mathrm{~min}$ of $2.5 \% \mathrm{CO}_{2}$ inhalation, the $\mathrm{PaCO}_{2}$ level increased to $74.1 \pm 7.1 \mathrm{mmHg}$, from 37.4 \pm 1.7 . At this time, LCBF, RBC concentration, and $\mathrm{RBC}$ velocity increased to $87.5 \pm 14.9$, $25.4 \pm 10.8$, and $41.5 \pm 7.9 \%$, respectively. After 20 min of $4.0 \% \mathrm{CO}_{2}$ inhalation, the $\mathrm{PaCO}_{2}$ level also increased to $92.5 \pm 6.2 \mathrm{mmHg}$, from $37.3 \pm 2.1$. LCBF, $\mathrm{RBC}$ concentration, and $\mathrm{RBC}$ velocity after $20 \mathrm{~min}$ of

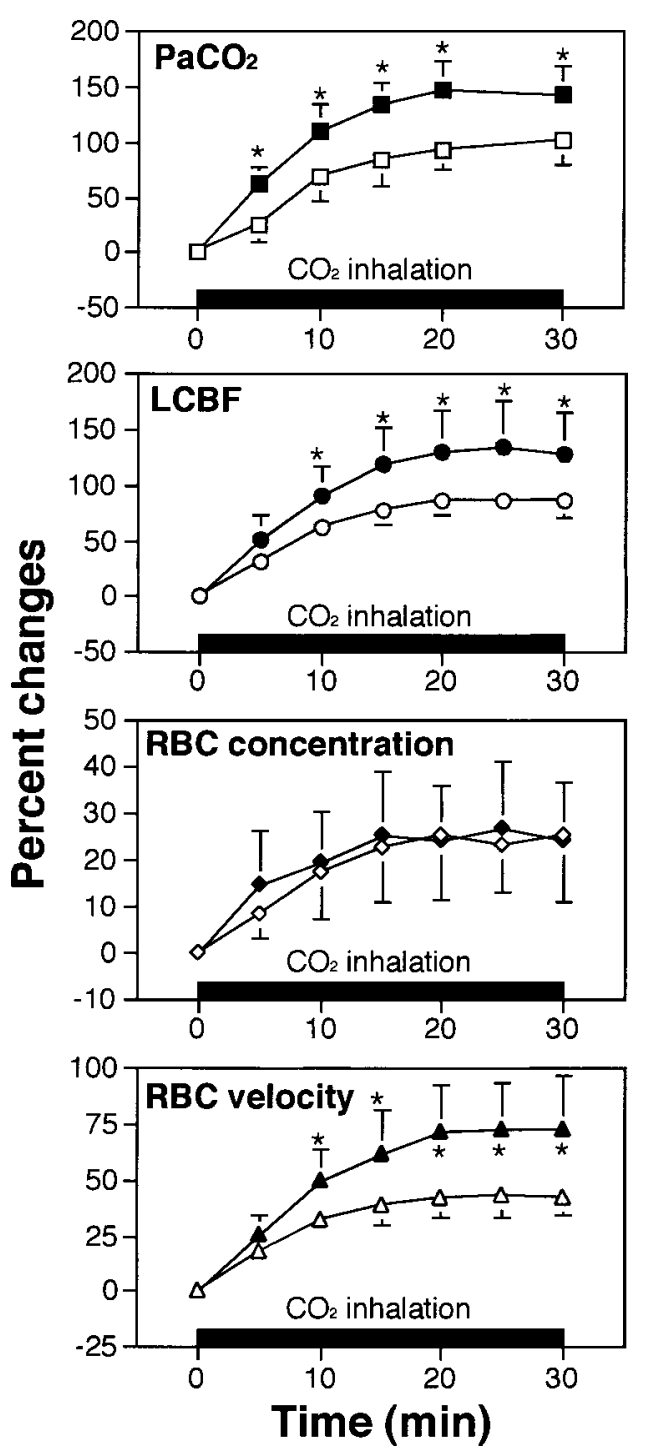

Fig. 1. The time course of changes in $\mathrm{PaCO}_{2}$ level, LCBF, RBC concentration, and RBC velocity in response to 2.5 and $4 \% \mathrm{CO}_{2}$ inhalation; values of the parameters obtained before $\mathrm{CO}_{2}$ inhalation (normocapnia) were considered as $\mathbf{0} \%$. Note that the increases in LCBF and $\mathrm{RBC}$ velocity during $4.0 \% \mathrm{CO}_{2}$ inhalation were higher than those during $2.5 \% \mathrm{CO}_{2}$ inhalation ( ${ }^{*} p<0.05$ ), though there was no significant difference in $\mathrm{RBC}$ concentration between the two $\mathrm{CO}_{2}$ conditions. Open and closed symbols indicate 2.5 and $4.0 \% \mathrm{CO}_{2}$ inhalation, respectively. Error bars indicate SD $(n=7)$.

$4.0 \% \mathrm{CO}_{2}$ inhalation increased to $130.0 \pm 38.2,24.3 \pm$ 13.0 , and $71.4 \pm 20.9 \%$, respectively. Under both $\mathrm{CO}_{2}$ conditions, changes in $\mathrm{RBC}$ velocity were higher than those in $\mathrm{RBC}$ concentration $(p<0.05)$.

The increases in LCBF and RBC velocity during $4.0 \% \mathrm{CO}_{2}$ inhalation were higher than those during $2.5 \% \mathrm{CO}_{2}$ inhalation $(p<0.05)$, although a significant difference after $5 \mathrm{~s}$ was not detected between the two conditions (Fig. 1). On the other hand, there was no significant difference in $\mathrm{RBC}$ concentration between 

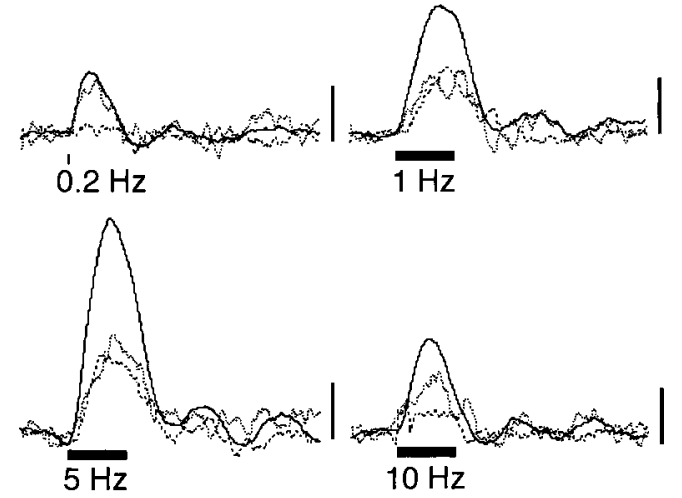

Fig. 2. Time response curves of evoked LCBF, RBC concentration, and RBC velocity at various frequencies for $\mathbf{5} \mathbf{s}$ stimulation of the hind paw. The response curves were normalized to the baseline level and averaged by the number of animals used $(n=14)$. Note that the responses of evoked LCBF, RBC concentration, and RBC velocity increased with increasing stimulus frequency up to $5 \mathrm{~Hz}$ and declined at $10 \mathrm{~Hz}$. Solid line: LCBF; fine dotted line: RBC concentration; rough dotted line: RBC velocity. Horizontal and vertical bars indicate the stimulation period and $5 \%$ increase with respect to the baseline level, respectively.

the conditions of 2.5 and $4.0 \% \mathrm{CO}_{2}$ inhalation.

\section{Changes in RBC behavior during neuronal activation (Study 2)}

The MABP and blood gas values in Study 2 were as follows $(n=14)$ : $\mathrm{MABP}=106.6 \pm 9.1 \mathrm{mmHg}, \mathrm{PaO}_{2}=$ $118.9 \pm 7.4 \mathrm{mmHg}$, and $P_{\mathrm{aCO}_{2}}=36.9 \pm 1.6 \mathrm{mmHg}$. These values were maintained within a stable range throughout the experiment. The arterial blood pressure did not change significantly during stimulation, which is consistent with our previous studies [8, 12].

The time response curves of the evoked LCBF to various stimulation frequencies are shown in Fig. 2. The evoked LCBF started to increase approximately $0.5 \mathrm{~s}$ after the onset of stimulation, regardless of the stimulus frequency. The response curve of evoked $\mathrm{LCBF}$ at 1,5 , and $10 \mathrm{~Hz}$ started to decline before the offset of stimulation, indicating that the peak value of evoked LCBF was noted before the cessation of neuronal activity. The evoked LCBF at $0.2 \mathrm{~Hz}$ peaked at $2.37 \pm 0.68 \mathrm{~s}$ and returned to the baseline level $4.79 \pm$ $0.82 \mathrm{~s}$ after the onset of stimulation. The evoked LCBF at 1 and $5 \mathrm{~Hz}$ peaked at $4.36 \pm 1.00$ and $4.00 \pm$ $0.62 \mathrm{~s}$ and returned to the baseline level $8.25 \pm 0.98$ and $8.45 \pm 0.80 \mathrm{~s}$, respectively, after the onset of stimulation. During $10 \mathrm{~Hz}$ stimulation, the evoked LCBF peaked at $3.05 \pm 0.58 \mathrm{~s}$ and returned to the baseline level $6.41 \pm 0.99 \mathrm{~s}$ after the onset of stimulation.

The response magnitudes of evoked LCBF, RBC concentration, and $\mathrm{RBC}$ velocity increased with increasing stimulus frequency up to $5 \mathrm{~Hz}$ and decreased

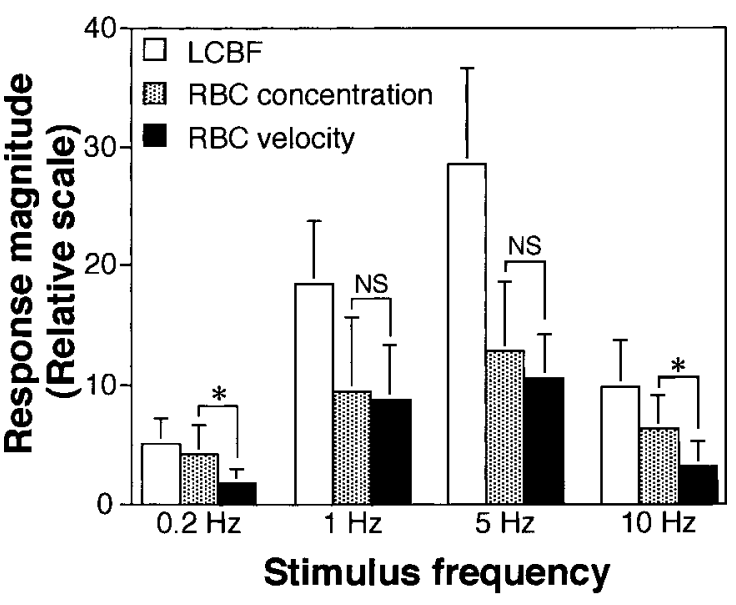

Fig. 3. A comparison of response magnitudes of evoked LCBF, RBC concentration, and RBC velocity. Normalized response magnitudes were calculated as an integral of the response curve of evoked LCBF from rise time to termination time (see details in text). Note that the response magnitudes of RBC concentration at 0.2 and $10 \mathrm{~Hz}$ were greater than those of $\mathrm{RBC}$ velocity $\left({ }^{*} p<0.05\right)$, though there were no significant differences in response magnitudes at 1 and $5 \mathrm{~Hz}$ stimulations between RBC concentration and RBC velocity. Error bars indicate SD $(n=14)$.

at $10 \mathrm{~Hz}$ (Figs. 2 and 3). However, the contributions of RBC concentration and $\mathrm{RBC}$ velocity to evoked LCBF at 0.2 and $10 \mathrm{~Hz}$ were not the same (Fig. 3). The response magnitudes of $\mathrm{RBC}$ concentration were greater than those of $\mathrm{RBC}$ velocity at 0.2 and $10 \mathrm{~Hz}$ $(p<0.05)$. On the other hand, at 1 and $5 \mathrm{~Hz}$ stimulation there was no significant difference in response magnitude between RBC concentration and RBC velocity (Fig. 3).

\section{DISCUSSION}

In this study, we demonstrated the independent dynamics of RBC concentration and $\mathrm{RBC}$ velocity by using LDF. It reflects only $\mathrm{RBC}$ behavior, which in the capillary bed is the main contributor to the LDF signal [10]. Thus the increase in RBC concentration within the capillary suggests an increase in the capillary volume, i.e., physiological recruitment [15] or capillary dilatation $[16,17]$, and the increase in RBC velocity at the capillary level is considered as being induced by an increase in transluminal pressure because of a decrease in the resistance of the upstream arteriole.

In the experiment of $\mathrm{CO}_{2}$ inhalation (Study 1), the MABP level after $30 \mathrm{~min}$ of $\mathrm{CO}_{2}$ inhalation decreased by approximately $10 \mathrm{mmHg}$ compared with that before $\mathrm{CO}_{2}$ inhalation $(p<0.05)$. This indicates that $\mathrm{CO}_{2}$ inhalation caused a decline in systemic perfusion 
pressure in this experiment. During $\mathrm{CO}_{2}$ inhalation, however, LCBF increased with an increase in RBC velocity in spite of a decrease in MABP level (Fig. 1). It is well known that cerebral blood flow (CBF) is maintained within a constant level by changes in vascular resistance even when MABP is slightly reduced (autoregulation), although an extreme change in blood pressure evoked by a strong stimulation of peripheral nerves, for example, affects CBF. These suggest that a decrease in perfusion pressure in the cortex is masked by a dilation of resistance vessels induced by $\mathrm{CO}_{2}$ inhalation (see below), or autoregulation is maintained even when the rat is subjected to $\alpha$-chloralose anesthesia.

Many reports have been made in which the relationship between $\mathrm{RBC}$ velocity and $\mathrm{RBC}$ concentration after $\mathrm{CO}_{2}$ inhalation was investigated. Most investigators observed an increase in RBC concentration besides an increase in $\mathrm{RBC}$ velocity during hypercapnia (e.g. $[10,16])$. An increase in RBC concentration during $\mathrm{CO}_{2}$ inhalation would indicate an increase in capillary volume under hypercapnia. Capillaries had been considered as rigid tubes. However, a moderate degree of distension of capillaries was observed by the microscopy of histological sections of rats subjected to hypercapnia in comparison with those subjected to hypocapnia [17], and an increase in capillary diameter was detected by confocal laser-scanning microscopy [16]. In the present study, we showed a larger change in $\mathrm{RBC}$ velocity than in $\mathrm{RBC}$ concentration during $\mathrm{CO}_{2}$ inhalation (Fig. 1). Moreover, the increase in $\mathrm{RBC}$ velocity during $4.0 \% \mathrm{CO}_{2}$ inhalation was higher than during $2.5 \% \mathrm{CO}_{2}$ inhalation $(p<0.05)$, and there was no significant difference in RBC concentration between the two conditions (Fig. 1). These suggest an increase in perfusion level in the capillary bed and a physical limitation of increase in capillary volume during hypercapnia. In both levels of $\mathrm{CO}_{2}$ inhalation, $\mathrm{RBC}$ concentration reached a peak (approximately a $25 \%$ increase) after $15 \mathrm{~min}$ of $\mathrm{CO}_{2}$ inhalation, and $\mathrm{LCBF}$ and $\mathrm{RBC}$ velocity continued to increase at this time and reached their peak after $20 \mathrm{~min}$ of $\mathrm{CO}_{2}$ inhalation (Fig. 1). These results indicate that the capillary volume would reach a maximal value when LCBF shows an increase of more than $75 \%$. This is consistent with results of a published study using LDF [10]. It is known that $\mathrm{pH}$ variations influence the tone of the vascular smooth muscle of resistance vessels [5]. Nitric oxide and adenosine, which are vasodilators, also play a role in this reactivity $[18,19]$. These suggest that a change in $\mathrm{PaCO}_{2}$ level causes a change in the production of these mediators, which are associated with the dilation of resistance vessels, causing a change in precapillary pressure and therefore a change in RBC velocity in the capillary bed.

Some possible mechanisms of LCBF regulation during neuronal activity are proposed. One mechanism is that the changes in LCBF are controlled only by resistance vessels, i.e., arterioles [6, 20, 21], and passive capillary changes may also be involved. Another possible mechanism proposed by Malonek et al. [7] is active capillary changes. In the present study we found that the responses of $\mathrm{RBC}$ concentration at 0.2 and $10 \mathrm{~Hz}$ were greater than those of $\mathrm{RBC}$ velocity $(p<0.05)$, though both RBC concentration and RBC velocity contributed equally at 1 and $5 \mathrm{~Hz}$ stimulation (Fig. 3). It has been known that neuronal activity in the somatosensory area would be maximum when the peripheral nerves are stimulated at approximately $5 \mathrm{~Hz}[4,6,8,11,22-24]$. This suggests that an increase in capillary volume is greater than in transluminal pressure when the activity of the somatosensory cortex is weak, and the contributions of these components become the same when the activity of the somatosensory area in response to peripheral stimulation reaches a maximal level. Based on our previous observation, we reported using direct electrical stimulation of the cortex that the increase in $\mathrm{RBC}$ velocity was larger than that in $\mathrm{RBC}$ concentration when the evoked LCBF reached a superabundant level [9]. These indicate that the contribution of capillary and resistance vessel would change in response to the level of evoked LCBF. In other words, the contribution of capillaries would be large during a small LCBF increase, and it would become smaller with an increasing level of evoked LCBF.

Adachi et al. demonstrated the effect of the stimulation of nucleus basalis of Meynert and hypercapnia on the pial artery diameter and change in LCBF, and described that hypercapnia caused an increase in the pial artery diameter and LCBF, but the stimulation of cholinergic fibers caused an increase in LCBF without a change in the pial artery diameter [25]. They also reported that evoked LCBF induced by cutaneous noxious stimulation was independent of the change in blood pressure and of any concomitant vasodilatation [26]. These observations support the concept of the neuronal control of evoked LCBF in the capillary level. In the present study, LCBF after $5 \mathrm{~min}$ of $2.5 \%$ $\mathrm{CO}_{2}$ inhalation showed a $30.2 \pm 10.4 \%$ increase compared with that before $\mathrm{CO}_{2}$ inhalation. This value was nearly the same as that of evoked LCBF at $5 \mathrm{~Hz}$. However, the contributions of $\mathrm{RBC}$ concentration and $\mathrm{RBC}$ velocity during somatosensory stimulation were different from those during $\mathrm{CO}_{2}$ inhalation. The increase in $\mathrm{RBC}$ concentration after $\mathrm{CO}_{2}$ inhalation was 
smaller than that in RBC velocity ( $p<0.05$; Fig. 1). On the other hand, there was no significant difference in response magnitude between $\mathrm{RBC}$ concentration and $\mathrm{RBC}$ velocity during somatosensory stimulation at $5 \mathrm{~Hz}$ (Fig. 3). These suggest that the increase in $\mathrm{LCBF}$ during neuronal activity is different from that of controlling the LCBF as induced by $\mathrm{CO}_{2}$. We conclude that the evoked LCBF is regulated by both capillary and arteriole changes and that the capillary and arteriole are controlled by independent mechanisms.

The authors are grateful to Mr. Yozo Ito of Akita Research Institute of Brain and Blood Vessels for technical assistance. Part of this study was supported by Japan Science and Technology Corporation (JST).

\section{REFERENCES}

1. Roy CS and Sherrington CS: On the regulation of the blood supply of the brain. J Physiol (Lond) 11: 85-108, 1980

2. Villringer $A$ and Dirnagl U: Coupling of brain activity and cerebral blood flow: basis of functional neuroimaging. Cerebrovasc Brain Metab Rev 7: 240-276, 1995

3. Matsuura T, Fujita H, Kashikura K, and Kanno I: Evoked local cerebral blood flow induced by somatosensory stimulation is proportional to the baseline flow. Neurosci Res 38: 341-348, 2000

4. Matsuura T and Kanno I: Quantitative and temporal relationship between local cerebral blood flow and neuronal activation induced by somatosensory stimulation in rats. Neurosci Res 40: 281-290, 2001

5. Lassen NA: Intrinsic regulation of blood flow in the brain. In: Peripheral Circulation, ed. Johnson PC, John Wiley and Sons Inc, New York, pp 337-358, 1978

6. Ngai AC, Meno JR, and Winn HR: Simultaneous measurements of pial arteriolar diameter and laser-Doppler flow during somatosensory stimulation. J Cereb Blood Flow Metab 15: 124-127, 1995

7. Malonek D, Dirnagl U, Lindauer U, Yamada K, Kanno I, and Grinvald A: Vascular imprints of neuronal activity: relationships between the dynamics of cortical blood flow, oxygenation, and volume changes following sensory stimulation. Proc Natl Acad Sci USA 94: 1482614831, 1997

8. Matsuura T, Fujita H, Seki C, Kashikura K, Yamada K, and Kanno I: CBF change evoked by somatosensory activation measured by laser-Doppler flowmetry: independent evaluation of RBC velocity and RBC concentration. Jpn J Physiol 49: 289-296, 1999

9. Matsuura T, Fujita H, Seki C, Kashikura K, and Kanno I: Hemodynamics evoked by microelectrical direct stimulation in rat somatosensory cortex. Comp Biochem Physiol A 124: 47-52, 1999

10. Barfod C, Akgören N, Fabriciu M, Dirnagl U, and Lauritzen M: Laser-Doppler measurements of concentration and velocity of moving blood cells in rat cerebral circulation. Acta Physiol Scand 160: 123-132, 1997
11. Detre JA, Ances BM, Takahashi K, and Greenberg JH: Signal averaged laser Doppler measurements of activation-flow coupling in the rat forepaw somatosensory cortex. Brain Res 796: 91-98, 1998

12. Matsuura T, Fujita H, Kashikura K, and Kanno I: Modulation of evoked cerebral blood flow under excessive blood supply and hyperoxic conditions. Jpn J Physiol 50: 115-123, 2000

13. Matsuura T, Kashikura K, and Kanno I: Hemodynamics of local cerebral blood flow induced by somatosensory stimulation under normoxia and hyperoxia in rats. Comp Biochem Physiol A 129: 363-372, 2001

14. Nilsson GE: Signal processor for laser Doppler tissue flowmeters. Med Biol Eng Comput 22: 343-348, 1984

15. Kuschinsky $W$ and Paulson OB: Capillary circulation in the brain. Cerebrovasc Brain Metab Rev 4: 261-286, 1992

16. Villringer $A$, Them $A$, Lindauer $U$, Einhäupl $K$, and Dirnagl U: Capillary perfusion of the rat brain cortex. An in vivo confocal microscopy study. Circ Res 75: 5562, 1994

17. Duelli $R$ and Kuschinsky W: Changes in brain capillary diameter during hypocapnia and hypercapnia. J Cereb Blood Flow Metab 13: 1025-1028, 1993

18. Estevez AY and Phillis JW: Hypercapnia-induced increases in cerebral blood flow: roles of adenosine, nitric oxide and cortical arousal. Brain Res 758: 1-8, 1997

19. Wang $Q$, Paulson $O B$, and Lassen NA: Effect of nitric oxide blockade by $N^{G}$-nitro-L-arginine on cerebral blood flow response to changes in carbon dioxide tension. J Cereb Blood Flow Metab 12: 947-953, 1992

20. Paulson $O B$ and Newman EA: Does the release of potassium from astrocyte endfeet regulate cerebral blood flow? Science 237: 896-898, 1987

21. Segal SS and Duling BR: Flow control among microvessels coordinated by intercellular conduction. Science 234: 868-870, 1986

22. Leniger-Follert $E$ and Hossmann KA: Simultaneous measurements of microflow and evoked potentials in the somatosensory cortex of the cat brain during specific sensory activation. Pflügers Arch 380: 85-89, 1979

23. Ngai AC, Jolley MA, D'Ambrosio R, Meno JR, and Winn HR: Frequency-dependent changes in cerebral blood flow and evoked potentials during somatosensory stimulation in the rat. Brain Res 837: 221-228, 1999

24. Silva AC, Lee S, Yang G, ladecola C, and Kim S: Simultaneous blood oxygenation level-dependent and cerebral blood flow function magnetic resonance imaging during forepaw stimulation in the rat. J Cereb Blood Flow Metab 19: 871-879, 1999

25. Adachi T, Baramidze DG, and Sato A: Stimulation of the nucleus basalis of Meynert increases cortical cerebral blood flow without influencing diameter of the pial artery in rats. Neurosci Lett 143: 173-176, 1992

26. Adachi T, Meguro K, Sato A, and Sato Y: Cutaneous stimulation regulates blood flow in cerebral cortex in anesthetized rats. Neuroreport 1: 41-44, 1990 